endotracheal intubation, all having received premedication; 5 infants had EEG suppression during endotracheal suctioning. Brain wave suppression was not correlated with SpO2, BP, heart rate, or TcCO2. (Shangle CE, Haas RH, Vaida F, Rich WD, Finer NN. Effects of endotracheal intubation and surfactant on a 3-channel neonatal electroencephalogram. J Pediatr 2012 Aug;161(2):252-7). (Reprints: Neil N Finer MD, UCSD, Department of Pediatrics, 200 West Arbor Dr, MPF 1-140, San Diego, CA 92103.E-mail: nfiner@ucsd.edu).

COMMENT. Endotracheal surfactant administration has reduced neonatal mortality and improved lung function in infants with respiratory distress syndrome. The adverse effects on neonatal brain electrical activity require further study of the EEG, using full neonatal electrode placement and including long-term outcome.

\title{
MRI IN HYPOXIC-ISCHEMIC ENCEPHALOPATHY
}

Researchers at University of Melbourne, Australia, and St Louis Children's Hospital, US investigated the effects of hypothermia treatment on MRI patterns of brain injury in newborns with hypoxic-ischemic encephalopathy (HIE) and the prognostic utility of MRI for death or disability at age 2 years. Fewer newborns had white matter and cortical gray matter abnormalities on T1- and T2-weighted images in the 66 treated with hypothermia group compared with 61 normothermic newborns. All T1- and T2weighted and diffusion MRI abnormalities were predictive of death or major sensorineural disability. The prognostic utility of MRI variables was not altered by hypothermia treatment. MRI abnormalities in the basal ganglia and thalami had the highest sensitivity and specificity for adverse outcome at 2 years. (Cheong JLY, Coleman L, Hunt RW, et al, for the Infant Cooling Evaluation Collaboration. Prognostic utility of magnetic resonance imaging in neonatal hypoxic-ischemic encephalopathy. Substudy of a randomized trial. Arch Pediatr Adolesc Med 2012 Jul 1;166(7):634-40). (Respond: Jeanie LY Cheong MD, Neonatal Service, Royal Women's Hospital, 20 Flemington Rd, Parkville 3052, Victoria, Australia (E-mail: Jeanie.cheong@thewomens.org.au).

COMMENT. Treatment with hypothermia is associated with reduction in white matter and cortical gray matter abnormalities on MRI of newborns with HIE. Abnormal MRI findings are prognostic of outcome in moderate to severe HIE in both hypothermia treated and normothermic newborns. MRI is an important biomarker of long-term outcome of newborns with HIE, irrespective of hypothermia.

The importance of timing of imaging and the greater sensitivity of some newer techniques are discussed in an editorial (Chau V et al. Magnetic resonance imaging in hypoxic-ischemic encephalopathy. Arch Pediatr Adolesc Med 2012 Jul 1;166(7):66971). In a previous study (Rutherford MA et al. Pediatrics 1998 Aug;102(2):323-8), MRI scans were done at a mean postnatal age of 8 days as opposed to 6 days in the present study, and the positive predictive value was somewhat lower (76\%). MR spectroscopy and diffusion tensor imaging will detect brain injury in the first days following an injury and may have better prognostic value than conventional MRI. (Porter EJ, et al. Tractbased spatial statistics of magnetic resonance images to assess disease and treatment effects in perinatal asphyxia encephalopathy. Pediatr Res 2010 Sep;68(3):205-9). 